\title{
Mental health of orthopaedic trauma patients during the 2020 COVID-19 pandemic
}

\author{
Erin Ohliger ${ }^{1}$ (I) Erica Umpierrez ${ }^{1} \cdot$ Lauren Buehler $^{2} \cdot$ Andrew W. Ohliger $^{3} \cdot$ Steven Magister $^{4} \cdot$ Heather Vallier $^{5}$. \\ Adam G. Hirschfeld ${ }^{5}$
}

Received: 4 June 2020 / Accepted: 6 July 2020 / Published online: 16 July 2020

(C) SICOT aisbl 2020

\begin{abstract}
Purpose The outbreak of the SARS-CoV-2 virus has been associated with reports of increased anxiety, depression and fear among the general population. People with underlying psychiatric disorders are more susceptible to stress than the general population. The purpose of this study was to determine the prevalence of concomitant psychiatric conditions in the orthopaedic trauma population during the COVID-19 pandemic.

Methods This retrospective cohort study evaluated orthopaedic trauma patients who received care at our institution between February through April of 2019 and February through April of 2020. Patient sex, age, mechanism of injury, associated injuries, fracture location, tobacco use, employment status, mental health diagnosis and presence of interpersonal violence were documented. Mental health diagnoses were defined based on International Classification of Diseases-10 classification.

Results The study included 553 orthopaedic patients evaluated at our institution during the defined time period. Patients in the 2020 cohort had a higher prevalence of mental health diagnoses $(26 \%$ vs. $43 \%, p<0.0001)$ compared with the 2019 group. The odds ratio for mental health disorder in the 2020 patients was 2.21 (95\% CI 1.54, 3.18) compared with the 2019 cohort. The 2020 cohort had a higher percentage of patients who reported interpersonal violence ( $20 \%$ vs. $11 \%, p=0.005)$.

Conclusion Our study showed a higher prevalence of psychiatric disease among orthopaedic trauma patients during the COVID19 pandemic when compared with those seen during the same time of the year in 2019. Stress induced by the coronavirus pandemic can place patients with mental illness at a higher risk for perilous behaviours and subsequent fractures.
\end{abstract}

Keywords COVID-19 $\cdot$ Orthopaedic trauma $\cdot$ Mental health $\cdot$ Interpersonal violence

Erin Ohliger

ohligee@ccf.org

1 Department of Orthopaedic Surgery, Cleveland Clinic, 9500 Euclid Avenue, Cleveland, $\mathrm{OH} 44195$, USA

2 Department of Endocrinology, Metabolism, and Diabetes, Cleveland Clinic, Cleveland, $\mathrm{OH}$, USA

3 Ohio University Heritage College of Osteopathic Medicine, Athens, OH, USA

4 Department of Orthopaedic Surgery, University Hospitals Case Medical Center, Cleveland, OH, USA

5 Department of Orthopaedic Surgery, MetroHealth Medical System, Cleveland, OH, USA

\section{Introduction}

The World Health Organization (WHO) declared coronavirus disease 2019 (COVID-19) a public health emergency of international concern on January 30, 2020 [1]. Given the high infectivity of SARS-CoV-2 and its rapid international spread, governments have enacted sweeping travel restrictions, quarantines and border shutdowns. The outbreak of this novel respiratory virus has been associated with reports of increased anxiety, depression, insomnia and fear among the general population $[2,3]$. In addition, extensive media coverage, stay at home orders and unprecedented unemployment rates have only intensified the sense of societal anxiety and panic [4].

Existing studies suggest that patients with psychiatric disorders are more susceptible to stress than the general population and may have heightened symptoms during a time of crisis [3-5]. In the USA, approximately $20 \%$ of adults experience mental illness [6]. Trauma patients represent a 
particularly vulnerable group as almost half of all hospitalized trauma patients in the USA have underlying psychiatric conditions [7-9]. The impact of psychiatric disease on orthopaedic problems and postoperative outcomes has been well established in the literature [10-13]. To our knowledge, there are no studies evaluating the effect of psychiatric disease on the rates of orthopaedic injury during a global pandemic.

During the COVID-19 crisis, more patients presented to our level 1 trauma centre with orthopaedic injuries related to domestic violence, substance abuse and self-injurious behaviour. The purpose of this study was to evaluate the prevalence of mental illness in patients presenting with new orthopaedic injuries during this time compared with patients seen in 2019. We hypothesized that there will be a more concomitant psychiatric conditions in the orthopaedic trauma population during the COVID-19 pandemic.

\section{Materials and methods}

\section{Study population}

After Institutional Review Board approval, a registry of trauma patients who received care at an urban level 1 trauma centre between February 1 through April 30 of 2019 and February 1 through April 30, 2020 was reviewed. Patients without orthopaedic injuries were excluded. Patient sex, age, mechanism of injury, associated injuries, fracture location (AO/Orthopaedic Trauma Association fracture classification), tobacco use, employment status, mental health diagnosis and presence of interpersonal violence were documented [14].

Mental health diagnoses were defined based on International Classification of Diseases-10 classification of mental and behavioural disorders from the World Health Organization [15]. For a diagnosis to be included, the patient had to have sought treatment for this condition by a mental health or primary care provider within the past 5 years. Interpersonal violence was classified as injuries resulting from blunt or penetrating assault or other domestic violence.

\section{Statistical analysis}

Categorical variables were summarized as frequencies and percentages, while continuous variables were described using means and standard deviations when normally distributed. Pearson chi-squared tests were used to compare categorical variables between the 2019 and 2020 patient cohorts. Odds ratios and $95 \%$ confidence intervals were calculated for report of interpersonal violence and history of mental health disorder. Student's $t$ test was used to compare continuous variables when normally distributed. All analyses were performed with a two-sided $p$ value $<0.05$ to determine statistical significance.
Source of funding The authors received no financial support for the research, authorship and/or publication of this article.

\section{Results}

Our study included 298 patients with orthopaedic injuries during 2019 and 255 patients during 2020. Age, sex, unemployment status, history of diabetes, tobacco smoking status and obesity were not significantly different between the two groups (Table 1). In 2019, there were 105 females (35\%) vs. 111 females $(44 \%)$ in the 2020 cohort $(p=0.46)$. Mean age was 52.1 (range 2-96) in 2019 vs. 48.8 (range 1-93 years) in 2020. Falls were the most common mechanism of injury in 2019 and 2020 (68\% vs. 71\%) followed by motor vehicle collisions (18.5\% vs. $12.9 \%)$ and gunshot wounds (5\% vs. $5.1 \%$ ) (Table 2).

In 2019, 76 patients (26\%) had a mental health diagnosis compared with 110 patients (43\%) in 2020. Patients in the 2020 cohort had more mental illness $(43 \%$ vs. $26 \%, p<$ 0.0001 ) compared with the 2019 group. The odds ratio for history of mental health disorder in the 2020 patients was 2.21 (95\% CI 1.54, 3.18) vs. the 2019 group. In 2019, 34 patients $(11 \%)$ reported interpersonal violence vs. 51 patients $(20 \%)$ in 2020. In 2020, more patients also reported interpersonal violence $(20 \%$ vs. $11 \%, p=0.005)$. The odds ratio of interpersonal violence was 1.94 (95\% CI 1.21, 3.12) (Table 3).

Mental health diagnoses in this patient cohort consisted of alcohol dependence, opioid dependence, cocaine dependence, social phobia, posttraumatic stress disorder, paranoid personality disorder, major depressive disorder, bipolar affective disorder, polysubstance abuse, schizoaffective disorder, schizophrenia, unspecified nonorganic psychosis, organic psychosis, anxiety, panic disorder, adjustment disorder, antisocial disorder, oppositional defiant disorder and borderline personality disorder. Frequencies of specific diagnoses by year are listed in Table 4 . There were notably higher rates of alcohol dependence ( $n=27$ vs. $n=7)$, depression ( $n=47$ vs. $n=34$ ), anxiety ( $n=21$ vs. $n=7)$ and people with multiple diagnoses $(n=$ 27 vs. $n=6$ ) in the 2020 cohort compared with 2019 patients (Table 4).

\section{Discussion}

The prevalence of mental health disorders in orthopaedic trauma patients was significantly higher during the 2020 COVID19 pandemic when compared with the same period of time the preceding year despite no changes to our local practice environment, population or referring hospital relationships. Interpersonal violence was significantly higher in the 2020 cohort suggesting that stressors due to the COVID-19 pandemic may be a factor. Furthermore, this study also found that 
Table 1 Comparison of 2019 and 2020 cohorts

\begin{tabular}{llll}
\hline & $2019(n=298)$ & $2020(n=255)$ & $p$ value \\
\hline Age, years (mean (SD)) & $52.1(23.6)$ & $48.8(23.5)$ & 0.08 \\
Female $(n(\%))$ & $105(35.2)$ & $111(43.5)$ & 0.046 \\
Unemployed $(n(\%))$ & $184(61.7)$ & $152(59.6)$ & 0.61 \\
Current smoker $(n(\%))$ & $115(38.6)$ & $103(40.4)$ & 0.67 \\
BMI $\geq 30 \mathrm{~kg} / \mathrm{m}^{2}(n(\%))$ & $76(25.5)$ & $60(23.5)$ & 0.59 \\
Diabetes $(n(\%))$ & $60(20.1)$ & $39(15.2)$ & 0.14 \\
Report of IPV $(n(\%))$ & $34(11.4)$ & $51(20.0)$ & 0.005 \\
Mental health diagnosis $(n(\%))$ & $76(25.5)$ & $110(43.1)$ & $<0.0001$ \\
\hline
\end{tabular}

$I P V$ interpersonal violence the proportion of women presenting with fracture was higher in the 2020 group. Prior studies have established that women are at high risk than men for vulnerability to interpersonal violence, which may explain this finding [16].

Healthcare crises such as pandemics have a profound psychological impact. Multiple studies have demonstrated that heightened stress responses during or following a crisis are associated with potentially long lasting adverse physical and mental health outcomes [2, 4, 17-20]. The outbreak of the Ebola virus in West Africa in 2014 received global media attention. Similar to the COVID-19 pandemic, widespread fear, panic and social isolation ensued throughout West Africa. High levels of anxiety, depression, adjustment disorders, posttraumatic stress disorder were seen not only patients with the Ebola virus but also members of the general public. As a result, following the resolution of the outbreak, mental health professionals recommended psychosocial interventions be included as part of future outbreak responses to combat the psychological stress associated with them [18, 19].

While necessary to decrease the spread of the virus, social isolation and quarantine may have contributed to psychological stress associated with the pandemic. Brooks et al. argue

Table 2 Mechanism of injury

\begin{tabular}{lll}
\hline & $2019(n=298)$ & $2020(n=255)$ \\
\hline Fall & $203(68.1 \%)$ & $181(71.0 \%)$ \\
MVC & $55(18.5 \%)$ & $33(12.9 \%)$ \\
MCC & $10(3.4 \%)$ & $7(2.7 \%)$ \\
GSW & $15(5.0 \%)$ & $13(5.1 \%)$ \\
Crush & $3(1.0 \%)$ & $7(2.7 \%)$ \\
Pedestrian vs. automobile & $8(2.7 \%)$ & $11(4.3 \%)$ \\
NAT & $0(0 \%)$ & $1(0.4 \%)$ \\
Unknown & $4(1.3 \%)$ & $2(0.8 \%)$ \\
\hline
\end{tabular}

$M V C$ motor vehicle collision, $M C C$ motor cycle collision, NAT nonaccidental trauma, GSW gunshot wound that inability to see loved ones, travel restrictions and boredom during isolation can have dramatic consequences [21]. Previous studies have reported that longer durations of quarantine result in worse mental health outcomes with higher rates of posttraumatic stress, avoidance behaviours and anger $[21,22]$. Furthermore, quarantine measures implemented during COVID-19 may also contribute to increased interpersonal violence in the form of domestic assault. Forced isolation in combination with the psychological and financial stress of the pandemic may increase risks for familial strife and partner abuse [18]. This is consistent with our work.

In the digital era, media consumption extends beyond broadcast networks to mobile devices and continuous social media access. The public relies heavily on the media as their source of information during times of crisis. However, excessive media coverage has been shown to add distress and panic [23]. Following the Boston Marathon bombings in 2013, Holman et al. found that exposure to over $6 \mathrm{~h}$ of bombingrelated media was associated with higher acute stress than direct exposure to the actual bombings [24]. Numerous studies have shown that the mental health problems associated with repeated exposure last for years beyond the coverage period $[24,25]$.

Patients with underlying mental health diagnoses may be more influenced by the fear, isolation and media coverage surrounding the COVID-19 pandemic. A heightened stress response relative to the general population may cause relapses or worsening of already existing mental health conditions. The stringent isolation orders and travel restrictions present additional challenges in establishing care, continuing treatment

Table 3 Odds ratios for IPV and mental health diagnoses

\begin{tabular}{lll}
\hline & Odds ratio & 95\% confidence interval \\
\hline Report of IPV & 1.94 & $(1.21,3.12)$ \\
Mental health diagnosis & 2.21 & $(1.54,3.18)$ \\
\hline
\end{tabular}

$I P V$ interpersonal violence 
Table 4 Type of mental health diagnoses

\begin{tabular}{lll}
\hline & 2019 & 2020 \\
\hline Alcohol dependence & 7 & 27 \\
Depression & 34 & 47 \\
Bipolar disorder & 10 & 11 \\
Opioid dependence & 5 & 4 \\
Anxiety & 7 & 21 \\
PTSD & 2 & 3 \\
Social phobia & 1 & 0 \\
Cocaine dependence & 0 & 1 \\
Schizophrenia & 5 & 6 \\
Schizoaffective disorder & 3 & 1 \\
Paranoid personality disorder & 2 & 0 \\
Polysubstance abuse & 3 & 7 \\
Organic psychosis & 0 & 3 \\
Nonspecified nonorganic psychosis & 1 & 0 \\
Borderline personality disorder & 1 & 1 \\
Antisocial disorder & 0 & 1 \\
Oppositional defiant disorder & 0 & 1 \\
Adjustment disorder & 0 & 3 \\
Obsessive compulsive disorder & 1 & 0 \\
Multiple diagnoses & 6 & 27 \\
Any diagnosis & 76 & 110 \\
\hline
\end{tabular}

and often even obtaining medications for this at-risk population [3].

Mental illness is seen at a higher proportion in trauma patients $[7,8]$, indicating that it may be a risk factor for orthopaedic injury, even in times of no pandemic. Our study showed a higher prevalence of psychiatric disease among the orthopaedic trauma patients seen at our institution during the COVID-19 pandemic when compared with those seen during the same time of the year in 2019. We also observed a higher rate of reported interpersonal violence in this year's patient cohort. Prior literature has shown that mental illness is associated with increased domestic violence, assault, substance abuse and self-injury, all of which are known risk factors for orthopaedic injury. Based on our results, it can be hypothesized that the stress induced by the COVID-19 pandemic can place patients with mental illness at a higher risk for these behaviours and therefore subsequent fractures.

Our study has several strengths and limitations. We examined a large sample at one institution using a control group of like patients with no COVID-19 exposure during the prior year. The retrospective nature of data collection may introduce reporting and selection biases. The ICD-10 codes to identify patients with mental illness may underrepresent the true prevalence due to discrepancies in provider documentation. Despite these limitations, we believe that our study provides novel and valuable insight into the impact of underlying mental health conditions on the orthopaedic trauma patients during the coronavirus pandemic. Increased provider awareness may improve recognition and treatment of patients with mental health and social needs beyond their orthopaedic injuries.

Data availability All data generated or analysed during this study are included in this published article (and its supplementary tables).

\section{Compliance with ethical standards}

Conflict of interest The authors declare that there is no conflict of interest.

Ethical approval Institutional review board approval was received for this retrospective review.

Consent to participate Not applicable to this retrospective review.

Consent for publication The authors and supporting institutions have consented to publication.

\section{References}

1. Sohrabi C, Alsafi Z, O'Neill N et al (2020) World Health Organization declares global emergency: a review of the 2019 novel coronavirus (COVID-19). Int J Surg 76:71-76

2. Kang L, Li Y, Hu S et al (2020) The mental health of medical workers in Wuhan, China dealing with the 2019 novel coronavirus. Lancet Psychiatry 7:14

3. Yao H, Chen JH, Xu YF, (2020) Patients with mental health disorders in the COVID-19 epidemic. Lancet Psychiatry, 7: p. 21

4. Rajkumar RP (2020) COVID-19 and mental health: a review of the existing literature. Asian J Psychiatr 52:102066

5. Chevance A, Gourion D, Hoertel N et al (2020) Ensuring mental health care during the SARS-CoV-2 epidemic in France: a narrative review. L'Encephale. https://doi.org/10.1016/j.encep.2020.03.001

6. Mental Health by Numbers. (2019) National Alliance of Mental Illness

7. Townsend LL, Esquivel MM, Uribe-Leitz T et al (2017) The prevalence of psychiatric diagnoses and associated mortality in hospitalized US trauma patients. J Surg Res 213:171-176

8. Weinberg DS, Narayanan AS, Boden KA et al (2016) Psychiatric illness is common among patients with orthopaedic polytrauma and is linked with poor outcomes. J Bone Joint Surg Am 98:341-348

9. Foster BD, Kang HP, Buser Z et al (2019) Effect of mental health conditions on complications, revision rates, and readmission rates following femoral shaft, tibial shaft, and pilon fracture. J Orthop Trauma 33:210-214

10. Carreon LY, Jespersen AB, Stottrup CC et al (2020) Is the hospital anxiety and depression scale associated with outcomes after lumbar spine surgery? Global Spine J 10:266-271

11. Utrillas-Compaired A, De la Torre-Escuredo BJ, Tebar-Martinez AJ et al (2014) Does preoperative psychologic distress influence pain, function, and quality of life after TKA? Clin Orthop Relat Res 472:2457-2465

12. Stubbs B, Perara G, Koyanagi A et al (2020) Risk of hospitalized falls and hip fractures in 22,103 older adults receiving mental health care vs 161,603 controls: a large cohort study. J Am Med Dir Assoc

13. Joseph NB, A. Flanagan, CD. Breslin, MA. Simpson, M. Ragone, C. Kalina, M. Hendrickson, S. Vallier, HA, (2020) Prevalence of 
posttraumatic stress disorder in acute trauma patients. OTA Int, 3: p. $56-58$

14. Meinberg EG, Agel J, Roberts CS et al (2018) Fracture and dislocation classification Compendium-2018. J Orthop Trauma 32(Suppl 1):s1-s170

15. (1994) The ICD-10 classification of mental and behavioural disorders: conversion tables between ICD-8, ICD-9 and ICD-10. World Health Organization. Division of Mental Health

16. Watts C, Zimmerman C (2002) Violence against women: global scope and magnitude. Lancet 359:1232-1237

17. Jeong H, Yim HW, Song YJ et al (2016) Mental health status of people isolated due to Middle East Respiratory Syndrome. Epidemiol Health 38:e2016048

18. Mohammed A, Sheikh TL, Poggensee G et al (2015) Mental health in emergency response: lessons from Ebola. Lancet Psychiatry 2: 955-957

19. Mohammed A, Sheikh TL, Gidado S et al (2015) An evaluation of psychological distress and social support of survivors and contacts of Ebola virus disease infection and their relatives in Lagos, Nigeria: a cross sectional study-2014. BMC Public Health 15:824

20. Shigemura J, Ursano RJ, Morganstein JC et al (2020) Public responses to the novel 2019 coronavirus (2019-nCoV) in Japan: mental health consequences and target populations. Psychiatry Clin Neurosci 74:281-282

21. Brooks SK, Webster RK, Smith LE et al (2020) The psychological impact of quarantine and how to reduce it: rapid review of the evidence. Lancet 395:912-920

22. Reynolds DL, Garay JR, Deamond SL et al (2008) Understanding, compliance and psychological impact of the SARS quarantine experience. Epidemiol Infect 136:997-1007

23. Thompson RR, Jones NM, Holman EA et al (2019) Media exposure to mass violence events can fuel a cycle of distress. Sci Adv 5: eaav3502

24. Holman EA, Garfin DR, Silver RC (2014) Media's role in broadcasting acute stress following the Boston Marathon bombings. Proc Natl Acad Sci U S A 111(p):93-98

25. Silver RC, Holman EA, Andersen JP et al (2013) Mental- and physical-health effects of acute exposure to media images of the September 11, 2001, attacks and the Iraq War. Psychol Sci 24: $1623-1634$

Publisher's note Springer Nature remains neutral with regard to jurisdictional claims in published maps and institutional affiliations. 